\title{
Integrable Boundaries and Universal TBA Functional Equations
}

\author{
C. H. Otto Chui, Christian Mercat and \\ Paul A. Pearce \\ Department of Mathematics and Statistics \\ University of Melbourne \\ Parkville, Victoria 3010, Australia
}

\begin{abstract}
We derive the fusion hierarchy of functional equations for critical $A-D-E$ lattice models related to the $s \ell(2)$ unitary minimal models, the parafermionic models and the supersymmetric models of conformal field theory and deduce the related TBA functional equations. The derivation uses fusion projectors and applies in the presence of all known integrable boundary conditions on the torus and cylinder. The resulting TBA functional equations are universal in the sense that they depend only on the Coxeter number of the $A-D-E$ graph and are independent of the particular integrable boundary conditions. We conjecture generally that TBA functional equations are universal for all integrable lattice models associated with rational CFTs and their integrable perturbations.
\end{abstract}

\section{Introduction}

Ever since Baxter solved [1] the eight-vertex model, commuting transfer matrix functional equations [2, 3, 4, 5, 6, 7] have been at the heart of the exact solution of two-dimensional lattice models on a periodic lattice by Yang-Baxter methods [8]. For theories such as the $A-D-E$ models considered here, these equations provide the key to obtaining free energies, correlation lengths and finite-size corrections. At criticality, the finite-size corrections are related to the central charges and scaling dimensions of the associated conformal field theory (CFT). Off-criticality, these corrections

1 C.Chui, C.Mercat, P.Pearce@ms.unimelb.edu.au 
yield the scaling energies of the associated (perturbed) integrable quantum field theory (QFT). The fundamental form of the functional equations involves fusion of the Boltzmann weights on the lattice and reflect the fusion rules of the associated CFT. However, in order to solve for finite-size corrections these functional equations need to be recast in the form of a $Y$-system or TBA functional equations [9, 6, 10]. Miraculously, it is then possible to solve [6] for the central charges and scaling dimensions using some special tricks and dilogarithm identities [11.

More recently, it has been realized [12, 13] that the Yang-Baxter methods and functional equations can be extended to systems in the presence of integrable boundaries on the cylinder by working with double row transfer matrices. It is then possible to calculate surface free energies and interfacial tensions [14] as well as finite-size corrections and conformal partition functions [15]. The critical $A-D-E$ models correspond, for different choices of regimes and/or fusion level, to unitary minimal models [16], parafermion theories [17] and superconformal theories [18]. The corresponding integrable boundary conditions on a cylinder can be constructed for each conformal boundary condition [19]. It is also possible to construct [20] integrable seams for each conformal twisted boundary condition [21] on the torus. In all such cases it should be possible to obtain the universal conformal properties in the continuum scaling limit by solving suitable functional equations.

In this paper we derive general fusion and TBA functional equations for the critical $A-D-E$ lattice models. Although the fusion hierarchy of functional equations is not universal, we show in this paper that the $Y$-system or TBA functional equations for the $A-D-E$ models are universal in the sense that they depend on the $A-D-E$ graph only through its Coxeter number, and more importantly, they are independent of the choice of integrable boundary conditions. For this reason no new miracles are required to solve for the universal conformal data of systems with conformal boundaries. In all cases the same functional equations must be solved! Instead, the different solutions required among the infinite number of possible solutions to these equations are selected by appropriate analyticity requirements.

The layout of the paper is as follows. We first recall some results about fused $A-D-E$ models in Sections 1.1 1.3. In Section 2, we define the transfer matrix for the different boundary conditions, on the torus and on the cylinder, with and without seams. In Section 3, we state the main result of the paper, that is the TBA equation, the boundary specific functional equations and their universal form. In Section 1 , we derive the equation. We first study the general idea which is based on local properties in 4.1 and 
we then apply it to the torus in 4.2 and the cylinder in 4.3 . We end up with a discussion in 5 .

\subsection{Face Weights}

A lattice model in the $A-D-E$ series is associated with a graph $G$, of $A$, $D$ or $E$ type. The spins are nodes of the graph $G$ and neighbouring sites on the lattice are allowed neighbouring nodes of the graph. The probability distribution of spins is defined by the critical (unfused) Boltzmann weight of each face (or plaquette) of spins, depending on a spectral parameter $u$ :

$$
W^{11}\left(\begin{array}{ll|l}
d & c & u \\
a & b & u
\end{array}\right)=\bigsqcup_{a}^{d} \underbrace{c}_{b}=s(\lambda-u) \delta_{a c}+s(u) \sqrt{\frac{\psi_{a} \psi_{c}}{\psi_{b} \psi_{d}}} \delta_{b d}
$$

where $g$ is the Coxeter number of $G, \lambda=\frac{\pi}{g}, s(u)=\frac{\sin (u)}{\sin (\lambda)}$ and $\psi_{a}$ is the entry of the Perron-Frobenius eigenvector of the adjacency matrix $G$, associated with the node $a$.

These Boltzmann weights are represented by a local face operator $X_{j}(u)$ in the Temperley-Lieb algebra $\mathcal{T}(N, \lambda)$ [16]:

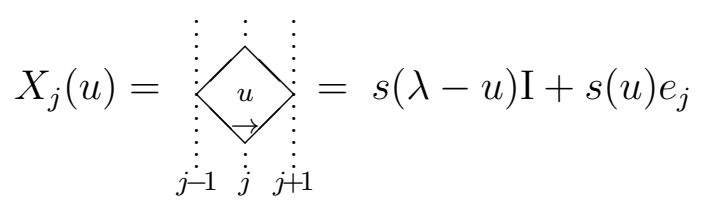

where $e_{j}=X_{j}(\lambda)$ is a Temperley-Lieb generator and $j$ is an integer labelling the $N$ faces of the lattice, assuming periodicity on the torus.

\subsection{Fusion Projector}

In turn, this model gives rise to a hierarchy of fused models whose Boltzmann weights we are going to describe.

We first define recursively the fusion operators $P_{j}^{r}$, for $r \in\langle 1, g\rangle$ as follows:

$$
\begin{aligned}
& P_{j}^{1}=P_{j}^{2}=I \\
& P_{j}^{r}=\frac{1}{S_{r-1}} P_{j+1}^{r-1} X_{j}(-(r-2) \lambda) P_{j+1}^{r-1}, \quad r \geq 3,
\end{aligned}
$$

where $S_{k}=s(k \lambda)$ and $j$ is clearly restricted in the cylinder case [16]. Thus, $P_{j}^{r}$ can be expressed as a function of $e_{j}, e_{j+1}, \ldots, e_{j+(r-3)}$. In particular,

$$
\left.P_{j}^{3}=\frac{1}{S_{2}}\langle-\lambda\rangle=I-\frac{1}{S_{2}} \measuredangle+\lambda\right\rangle .
$$


We shall represent the fusion operators diagrammatically as

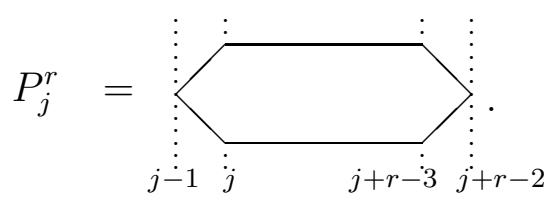

It is easy to show that this operator is in fact a projector. Moreover,

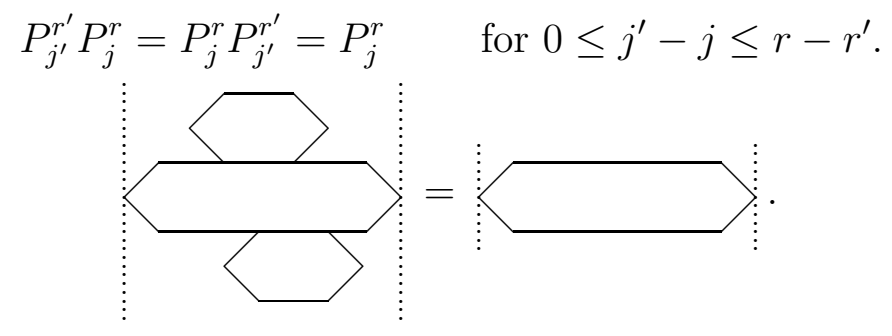

This implies that any operator expressible as a product of local face operators and falling within the boundaries of a projector, acts as a scalar on it:

$$
X_{j^{\prime}}(u) P_{j}^{r}=P_{j}^{r} X_{j^{\prime}}(u)=s(\lambda-u) P_{j}^{r} \quad \text { for } 0 \leq j^{\prime}-j \leq r-2 .
$$

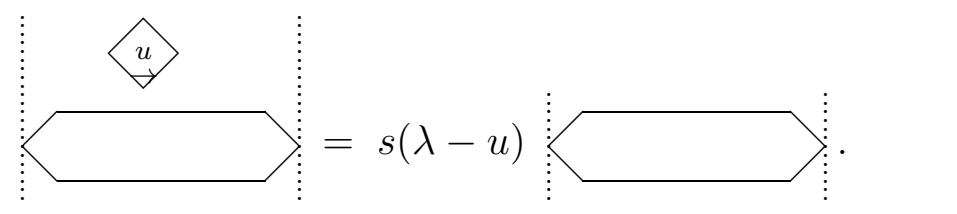

A particularly important case is for $u=+\lambda$ : its local face operator is a projector orthogonal to all the $P_{j}^{r}$.

As $P_{j}^{r}$ is clearly translationally covariant (in its domain of definition) we can decompose it onto the spaces of paths with given end points: $P^{r}(a, b)$ is the fusion projector acting on paths from $a$ to $b$ in $r-1$ steps. Its rank is given by the fused adjacency matrix entries:

$$
\operatorname{Rank}\left(P^{r}(a, b)\right)=F_{a b}^{r}
$$

also called basic intertwiners and recursively defined by the $\hat{s \ell_{2}}$ fusion rules:

$$
F^{1}=\mathrm{I}, \quad F^{2}=G, \quad F^{r}=F^{r-1} F^{2}-F^{r-2}, \text { for } r=3, \ldots, g .
$$

The +1 eigenvectors of $P^{r}(a, b)$ are thus indexed by an integer $\gamma \in\left\langle 1, F_{a b}^{r}\right\rangle$ refered to as the bond variable. We denote them $\boldsymbol{U}_{\gamma}^{r}(a, b)$, the fusion vectors. 


\subsection{Fused face operators}

These projectors allow us to define the $(p, q)$-fused face operator whose main feature is the product of $q$ rows of $p$ local face operators with a shift of the spectral parameter by $\pm \lambda$ from one face to the next:

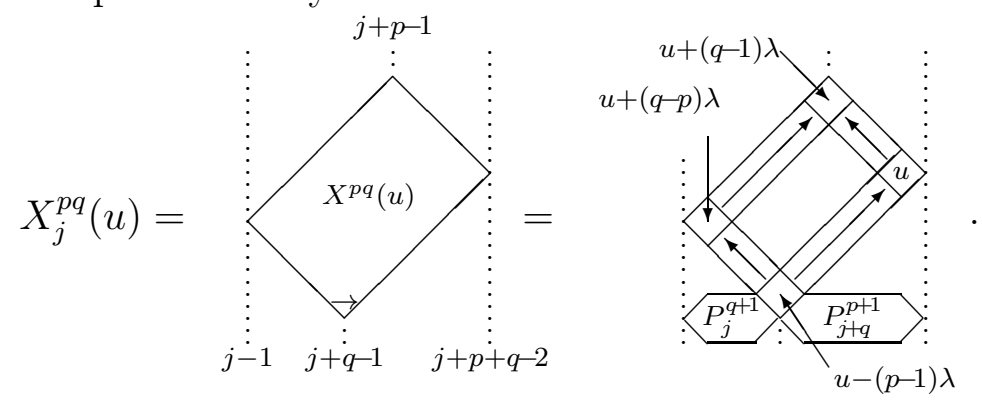

The position of the projectors and spectral parameters can be altered by pushing-through:

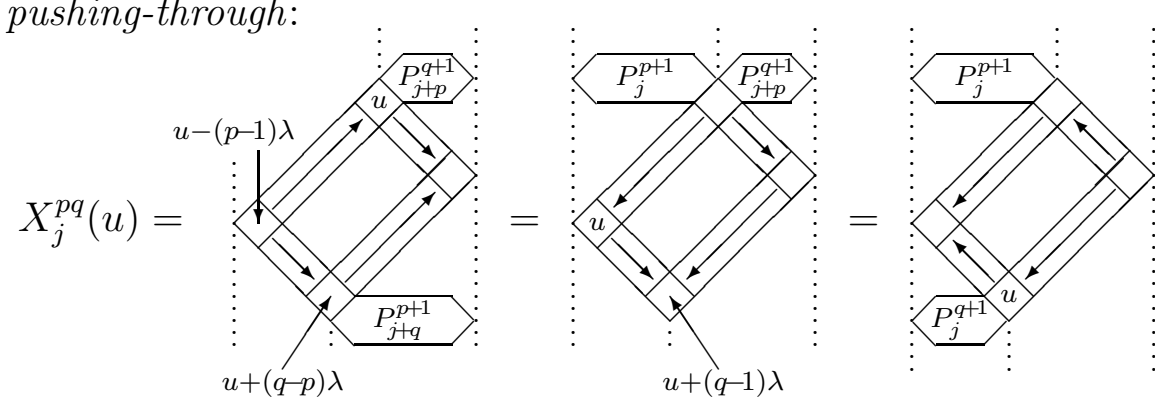

These properties imply several others, namely the Transposition Symmetry,

$$
X_{j}^{p q}(u)^{T}=X_{j}^{q p}(u+(q-p) \lambda),
$$

the Generalized Yang-Baxter Equation (GYBE),

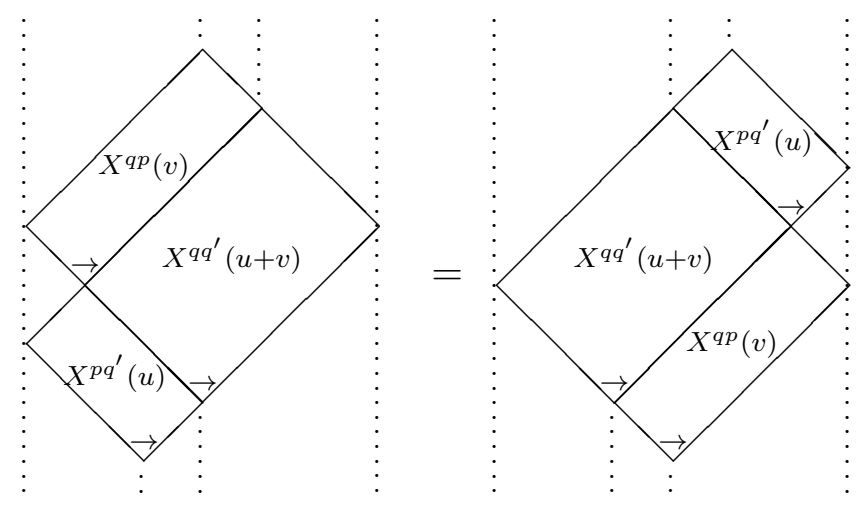

the Inversion Relation,

$$
\begin{gathered}
X_{j}^{p q}(u) X_{j}^{q p}(-u)=\ldots \\
=s_{1}^{p q}(u) s_{1}^{p q}(-u) P_{j}^{q+1} P_{j+q}^{p+1}
\end{gathered}
$$


where $s_{i}^{p q}(u)=\prod_{j=0}^{q-1} \prod_{k=0}^{p-1} s(u+(i-j+k) \lambda)$, and the Abelian Property,

$$
X_{j}^{p q}(u+(p-1) \lambda) X_{j}^{q p}(v+(q-1) \lambda)=X_{j}^{p q}(v+(p-1) \lambda) X_{j}^{q p}(u+(q-1) \lambda) .
$$

These operators, contracted against the fusion vectors, yield the $(p, q)$ fused Boltzmann weight. It depends not only on the spins at its four corners but also on bond variables on its edges:

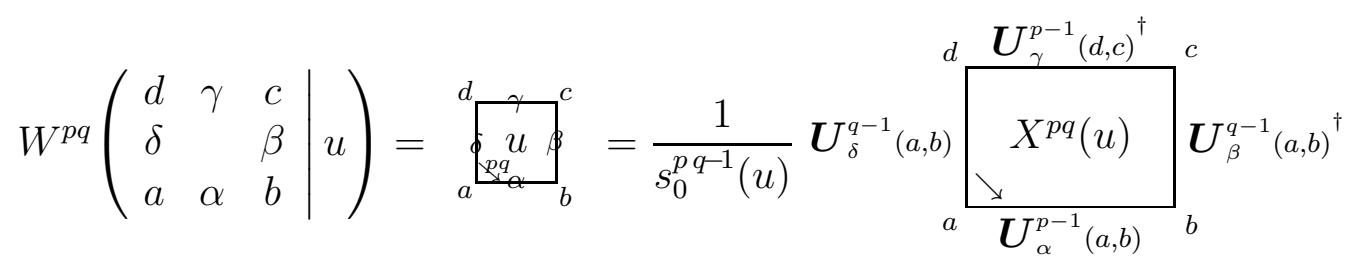

where the function $s_{0}^{p q-1}(u)$ eliminates some scalar factors common to all the spin configurations which appear in the process of fusion. In the $A_{L}$ case, the bond variables are trivial.

These fused Boltzmann weights satisfy the following symmetry

$$
\left.W^{p q}\left(\begin{array}{ccc}
d & \gamma & c \\
\delta & & \beta \\
a & \alpha & b
\end{array}\right) u\right)=\frac{s_{q-p}^{q p-1}(u)}{s_{0}^{p q-1}(u)} W^{q p}\left(\begin{array}{ccc}
d & \delta & a \\
\gamma & & \alpha \\
c & \beta & b
\end{array} \mid u+(q-p) \lambda\right)
$$

and Crossing Symmetry:

$$
W^{p q}\left(\begin{array}{ccc|}
d & \gamma & c \\
\delta & & \beta \\
a & \alpha & b
\end{array} \mid u\right)=\sqrt{\frac{\psi_{a} \psi_{c}}{\psi_{b} \psi_{d}}} \frac{s_{0}^{q p-1}(\lambda-u)}{s_{0}^{p q-1}(u)} W^{q p}\left(\begin{array}{ccc}
a & \delta & d \\
\alpha & & \gamma \\
b & \beta & c
\end{array} \mid \lambda-u\right) .
$$

\section{Transfer matrix}

Given this fusion hierarchy, we build transfer matrices for different fusion levels and boundary conditions: on the torus, and on the cylinder, with or without seams.

\subsection{Seam}

Simple seams are modified faces. They come in three different types, $r, s$ and $\zeta$-type. A label $(r, s, \zeta) \in A_{g-2} \times A_{g-1} \times \Gamma$, where $\Gamma$ is the symmetry 
algebra of the graph $G$, encodes a triple seam involving three modified faces. The symmetry $\zeta$ is taken as the identity when omitted.

We first define $W_{(r, 1)}^{q}$, the $r$-type seam for the $(p, q)$-fused model. It is a usual $(r-1, q)$-fused face (it doesn't depend on the horizontal fusion level $p)$ with an extra parameter $\xi$ acting as a shift in the spectral parameter, and another choice for the removal of the common scalar factors:

$W_{(r, 1)}^{q}\left(\begin{array}{ccc}d & \gamma & c \\ \delta & & \beta \\ a & \alpha & b\end{array} \mid u, \xi\right)=\underbrace{d \alpha_{b}(u, \xi)}_{a \alpha_{a}}]_{b}^{c}=\frac{s_{0}^{r-1 q-1}(u+\xi)}{s_{1}^{r-2 q}(u+\xi)} W^{(r-1) q}\left(\begin{array}{ccc|c}d & \gamma & c \\ \delta & & \beta \\ a & \alpha & b\end{array} \mid u+\xi\right)$.

An $s$-type seam is the normalized braid limit of an $r$-type seam, it doesn't depend on any spectral parameter:

$W_{(1, s)}^{q}\left(\begin{array}{ccc}d & \gamma & c \\ \delta & & \beta \\ a & \alpha & b\end{array}\right)=\underbrace{\left.d(1, s)\right|_{b} ^{c}}_{a}=\lim _{\xi \rightarrow i \infty} \frac{e^{-i \frac{(g+1)(s-1) q}{2} \lambda}}{s_{0}^{1 q}(u+\xi)} W_{(s, 1)}^{q}\left(\begin{array}{ccc}d & \gamma & c \\ \delta & & \beta \\ a & \alpha & b\end{array} \mid u, \xi\right)$.

The automorphisms $\zeta \in \Gamma$ of the adjacency matrix, satisfying $G_{a, b}=$ $G_{\zeta(a), \zeta(b)}$, leave the face weights invariant

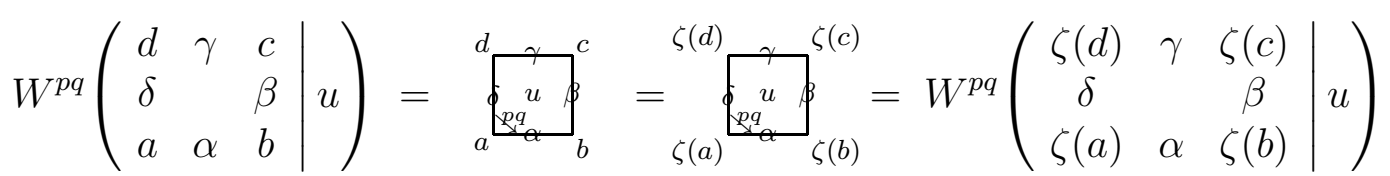

and act through the special seam [22]

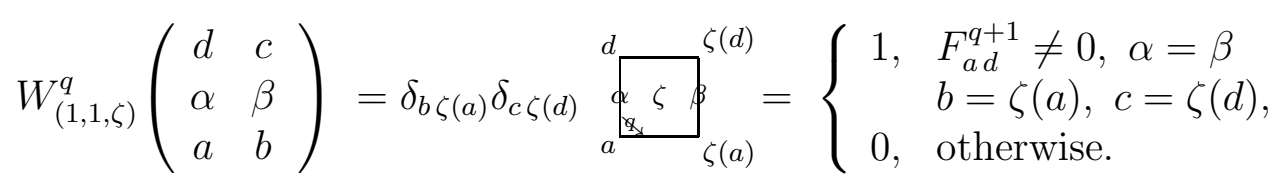

Notice that the $(r, s, \zeta)=(1,1,1)$ seam, where $\zeta=1$ denotes the identity automorphism, is the empty seam

$$
W_{(1,1,1)}^{q}\left(\begin{array}{cc}
d & c \\
\alpha & \beta \\
a & b
\end{array}\right)=\delta_{a b} \delta_{c d} \delta_{\alpha \beta} F_{b c}^{q+1}
$$

The push-through property is also trivially verified for a $\zeta$-type seam.

The label $s$ appearing in a $(1, s)$-seam is an integer in $A_{g-1}$. In [23], we define an $(1, a)$-seam with $a \in G$ which reduces to the definition given here for $G$ of $A$ type but which extends it for the $D_{\text {even }}, E_{6}$ and $E_{8}$ graphs. 


\subsection{Torus transfer matrix}

The transfer matrix for the $(p, q)$-fused model with an $(r, s, \zeta)$-seam, on the $N$ faces torus on the square lattice is given, in the basis of the cyclic paths in $N$ steps plus the seam, with bond variables between adjacent spins, by the product of the corresponding Boltzmann weights: The entries of the transfer matrix with an $(r, s, \zeta)$ seam are given by

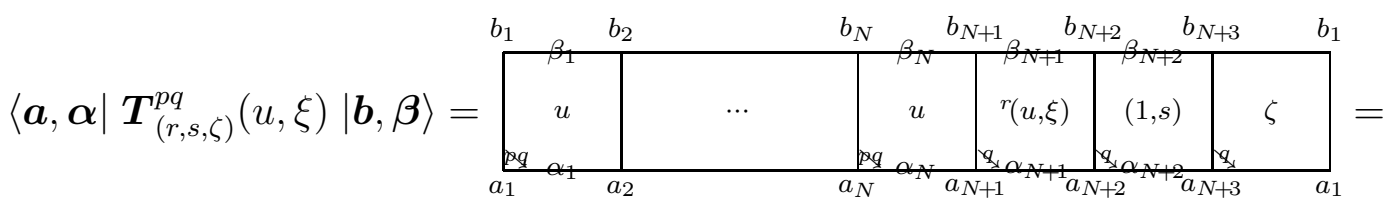

$$
\begin{aligned}
& \sum_{\gamma} \prod_{i=1}^{N} W^{p q}\left(\begin{array}{ccc}
b_{i} & \beta_{i} & b_{i+1} \\
\gamma_{i} & & \gamma_{i+1} \\
a_{i} & \alpha_{i} & a_{i+1}
\end{array} \mid u\right) W_{(r, 1)}^{q}\left(\begin{array}{ccc}
b_{N} & \beta_{N} & b_{N+1} \\
\gamma_{N} & & \gamma_{N+1} \\
a_{N} & \alpha_{N} & a_{N+1}
\end{array} \mid u, \xi\right) \times \\
& W_{(1, s)}^{q}\left(\begin{array}{ccc}
b_{N+1} & \beta_{N+1} & b_{N+2} \\
\gamma_{N+1} & & \gamma_{N+2} \\
a_{N+1} & \alpha_{N+1} & a_{N+2}
\end{array}\right) W_{(1,1, \zeta)}^{q}\left(\begin{array}{cc}
b_{N+2} & b_{1} \\
\gamma_{N+2} & \gamma_{1} \\
a_{N+2} & a_{1}
\end{array}\right)
\end{aligned}
$$

where the sum is over all possible vertical bond variables. The usual periodic boundary condition is obtained for $(r, s, \zeta)=(1,1,1)$. The definition can be generalised to accomodate an arbitrary number of seams. Because the seam faces are modified bulk faces, they satisfy the GYBE, so they can be moved around freely with respect to the bulk faces, the spectrum of the corresponding transfer matrices remains unchanged. However, in the $D_{2 k}$ cases, when there are several seams, their order can not be exchanged because the fusion algebra of defect lines is non commutative [24, 23].

\subsection{Boundary weights}

The boundary weights are labelled by $(r, a)$ with $r \in A_{g-2}$ a fusion level and $a \in G$ a node of the graph.

In the $A_{L}$ case, all $(r, s)$ boundary weights are obtained from the action of an $(r, s)$-seam on the vacuum boundary weight [25] and we will construct in [23] an $(r, a)$-seam with $a \in G$ so that it is also the case for the $D_{\text {even }}, E_{6}$ and $E_{8}$ graphs. Nevertheless, in all cases, the $(1, a)$ boundary weights, for two $q$-adjacent nodes of $G, c$ and $a$ (i.e. $F_{a c}^{q+1} \neq 0$ ) are given explicitely by

$$
B_{(1, a)}^{q}\left(\begin{array}{c}
\gamma^{a} \\
c_{\alpha}
\end{array}\right)=c\left\langle(1, a)=\frac{\psi_{c}^{1 / 2}}{\psi_{a}^{1 / 2}} \boldsymbol{U}_{\gamma}^{q+1}(c, a)^{\dagger} \boldsymbol{U}_{\alpha}^{q+1}(c, a)=\frac{\psi_{c}^{1 / 2}}{\psi_{a}^{1 / 2}} \delta_{\gamma \alpha} .\right.
$$


The vacuum boundary condition usually corresponds to $(1, a)=(1,1)$. The full $(r, a)$ boundary weights are then given by the action of an $r$-type seam onto the $(1, a)$-boundary weight. The double row seam is given by two regular $r$-seams sharing the same extra spectral parameter $\xi$, placed on top of one another, with the same spectral parameters as bulk faces appearing in the double row transfer matrix defined below in (1.29):

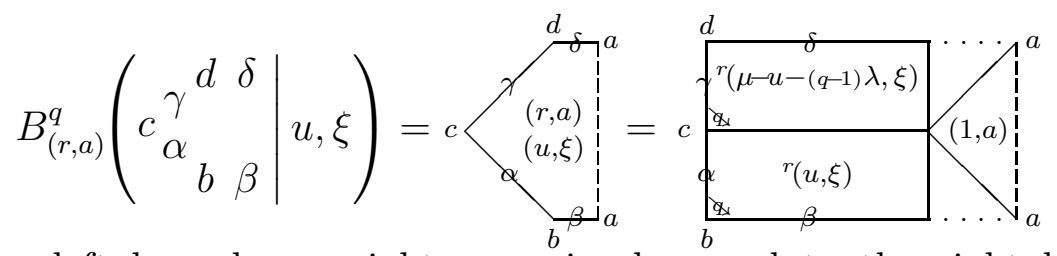

and the left boundary weights are simply equal to the right boundary weights.

These boundary weights satisfy boundary versions of the equations the bulk faces satisfy. The Generalized Boundary Yang-Baxter Equation or reflection equation is

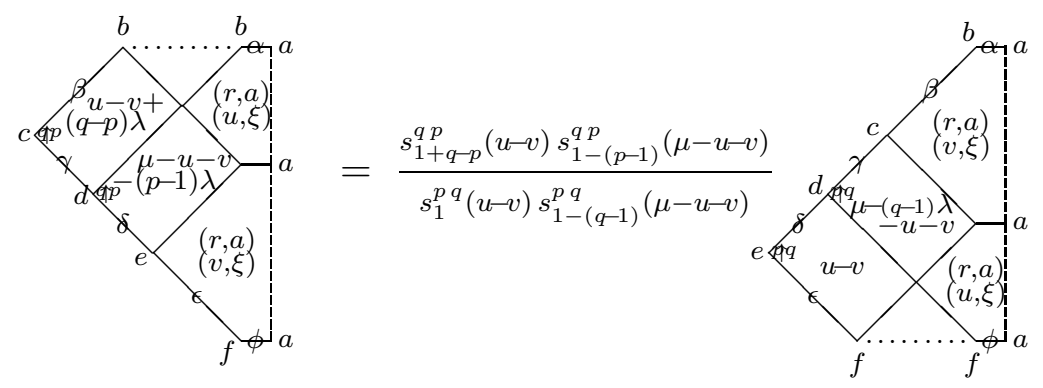

which is proved using the GYBE (1.13) and the abelian property (1.15).

We refer to [13, 16] for the boundary crossing equation.

Let's state here a property that will be of use later on. By Equation (1.7), one can fill up the triangle appearing in the definition (1.25) of the $(1, a)$ boundary weight with any local face operators: they will only contribute through a scalar factor, hence,

$$
B_{(1, a)}^{q}\left(\begin{array}{c}
\gamma^{a} \\
c_{a}
\end{array}\right)=\frac{\psi_{c}^{1 / 2}}{\psi_{a}^{1 / 2}} \prod_{i=1}^{q-1} \frac{1}{s_{1-i}^{i 1}(-2 u)}{ }_{2 u+(q-1)} \vdots_{U_{\alpha}^{q+1}(c, a)}^{q+1}(c, a)^{\dagger}
$$

\footnotetext{
${ }^{2}$ When extra structure is imposed, like in the superconformal case [18], the
} vacuum of the problem can be more complicated. 


\subsection{Double row transfer matrix}

The double row transfer matrix is given by two rows similar to the one appearing in the torus transfer matrix, with spectral parameters $u$ for the bottom one and $\mu-u-(q-1) \lambda$ for the top one, where $\mu$ is a fixed parameter and $q$ is the vertical fusion level. The boundary condition is not cyclic but given by the boundary weights defined previously (1.26).

$$
\left\langle\boldsymbol{a}, \boldsymbol{\alpha}\left|\boldsymbol{T}_{\left(r_{L}, a_{L}\right)|(r, s, \zeta)|\left(r_{R}, a_{R}\right)}^{p q}\left(u, \xi_{L}, \xi, \xi_{R}\right)\right| \boldsymbol{b}, \boldsymbol{\beta}\right\rangle=
$$

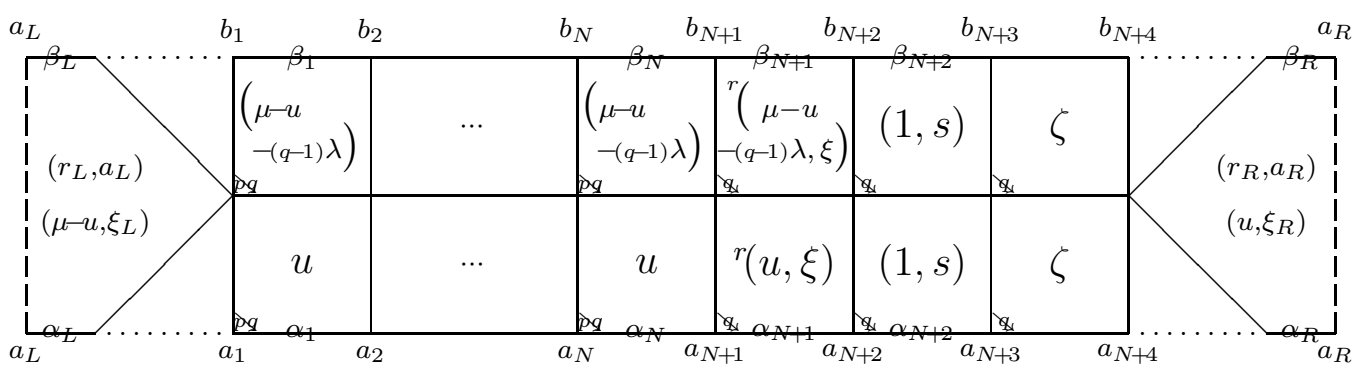

The GYBE (1.13) implies that double row transfer matrices with the same boundary conditions and boundary fields commute:

$$
\begin{aligned}
& \boldsymbol{T}_{\left(r_{L}, a_{L}\right)|(r, s, \zeta)|\left(r_{R}, a_{R}\right)}^{p q}\left(u, \xi_{L}, \xi, \xi_{R}\right) \boldsymbol{T}_{\left(r_{L}, a_{L}\right)|(r, s, \zeta)|\left(r_{R}, a_{R}\right)}^{p q^{\prime}}\left(v, \xi_{L}, \xi, \xi_{R}\right)= \\
& \boldsymbol{T}_{\left(r_{L}, a_{L}\right)|(r, s, \zeta)|\left(r_{R}, a_{R}\right)}^{p q^{\prime}}\left(v, \xi_{L}, \xi, \xi_{R}\right) \boldsymbol{T}_{\left(r_{L}, a_{L}\right)|(r, s, \zeta)|\left(r_{R}, a_{R}\right)}^{p q}\left(u, \xi_{L}, \xi, \xi_{R}\right) .
\end{aligned}
$$

\section{Fusion hierachies}

These transfer matrices fulfill a fusion hierarchy of functional equations. The details of these equations do depend on the type of matrices but their structure is the same. It stems from local properties that they all satisfy. Let's choose a horizontal fusion level $p$, and a fixed boundary condition among those available, namely toroidal, cylindrical, with or without seams. Call $\boldsymbol{T}_{k}^{q}(u)$ the corresponding $(p, q)$-fused transfer matrix at spectral parameter $u+k \lambda$, for $-1 \leq q \leq g-3$, with $\boldsymbol{T}_{0}^{-1}$ and $\boldsymbol{T}_{0}^{0}$ defined as

$$
\boldsymbol{T}_{0}^{-1}=\mathbf{0}, \quad \boldsymbol{T}_{0}^{0}=f_{-1}^{p} \boldsymbol{I} ;
$$

where $f_{q}^{p}$ is the usual order- $N$ bulk term

$$
f_{q}^{p}(u)= \begin{cases}{\left[s_{q}^{p}(u)\right]^{N},} & \text { for the torus } \\ (-1)^{p N}\left[s_{q}^{p}(u) s_{q+p}^{p}(u-\mu)\right]^{N}, & \text { for the cylinder. }\end{cases}
$$


Then the matrices $\left(\boldsymbol{T}^{q}\right)_{-1 \leq q \leq g-3}$ fulfill a hierarchy of functional equations

$$
\boldsymbol{T}_{0}^{q} \boldsymbol{T}_{q}^{1}=V_{q} \Phi_{q} f_{q}^{p} \boldsymbol{T}_{0}^{q-1}+\tilde{V}_{q} f_{q-1}^{p} \boldsymbol{T}_{0}^{q+1}
$$

where $f_{q}^{p}, \Phi_{q}, V_{q}$ and $\tilde{V}_{q}$, are (scalar) functions that we are going to describe, contributions of the bulk faces, the seams and the cylindrical $(1, a)$ boundary conditions respectively.

We have just given the form of the bulk free energy (1.32).

The functions $V_{q}$ and $\tilde{V}_{q}$ are trivial in the torus case, $V_{q}=\tilde{V}_{q}=1$, and on the cylinder, they are given by

$$
V_{q}=\frac{s_{q-2}(2 u-\mu) s_{2 q+1}(2 u-\mu)}{s_{q-1}(2 u-\mu) s_{2 q}(2 u-\mu)},
$$

and

$$
\tilde{V}_{q}=\frac{s_{q}(2 u-\mu) s_{2 q-1}(2 u-\mu)}{s_{q-1}(2 u-\mu) s_{2 q}(2 u-\mu)} .
$$

The function $\Phi_{q}$ is the product of order-1 terms coming from the seams. As we saw in Section 2.3, an $(r, a)$-boundary condition is constructed from an $r$-seam on a $(1, a)$-boundary condition, and we do count as separate type $r$ seams the ones coming from the left and from the right boundaries. If there are $K$ seams, the function $\Phi_{q}$ is given by a product of $K$ similar terms:

$$
\Phi_{q}=\prod_{k=1}^{K} \phi_{q}\left(r_{k}, \xi_{k}, u\right)
$$

The contribution of an $(r, s, \zeta)$-seam only depends on $r$ and $\phi_{q}(1, \xi, u)=1$. For $2 \leq r \leq g-2$,

$$
\phi_{q}(r, \xi, u)= \begin{cases}\phi_{q}^{\mathrm{t}}(r, \xi, u)=s_{q-r}(u+\xi) s_{q}(u+\xi), & \text { for the torus } \\ \phi_{q}^{\mathrm{t}}(r, \xi, u) \phi_{q+r-1}^{\mathrm{t}}(r, \mu+\xi, u), & \text { for the cylinder. }\end{cases}
$$

More generally, we have the following hierarchy of inversion identities which can be proved by induction as done in [6]:

$$
A_{q} \boldsymbol{T}_{0}^{q} \boldsymbol{T}_{1}^{q}=B_{q} f_{-1}^{p} f_{q}^{p} \prod_{k=1}^{q} \Phi_{k} \boldsymbol{I}+C_{q} \boldsymbol{T}_{0}^{q+1} \boldsymbol{T}_{1}^{q-1}
$$

where, in the torus case the functions $A_{q}=B_{q}=C_{q}=1$ are trivial, and

$$
A_{q}(u)=s_{q-1}(2 u-\mu) s_{q+1}(2 u-\mu)
$$




$$
\begin{gathered}
B_{q}(u)=s_{-1}(2 u-\mu) s_{2 q+1}(2 u-\mu), \\
C_{q}(u)=\left[s_{q}(2 u-\mu)\right]^{2},
\end{gathered}
$$

are due to the left and right vacuum boundaries in the cylinder case.

If we further define the normalised transfer matrices

$$
\boldsymbol{t}_{0}^{q}=\frac{C_{q} \boldsymbol{T}_{1}^{q-1} \boldsymbol{T}_{0}^{q+1}}{B_{q} f_{-1}^{p} f_{q}^{p} \prod_{k=1}^{q} \Phi_{k}},
$$

then the inversion identity hierarchy can be recast in the form of the following universal thermodynamic Bethe ansatz (TBA) functional equation

$$
\boldsymbol{t}_{0}^{q} \boldsymbol{t}_{1}^{q}=\left(\boldsymbol{I}+\boldsymbol{t}_{1}^{q-1}\right)\left(\boldsymbol{I}+\boldsymbol{t}_{0}^{q+1}\right)
$$

In deriving the TBA equation we have used the simple properties

$$
B_{q}(u) B_{q}(u+\lambda)=B_{q-1}(u+\lambda) B_{q+1}(u),
$$

and

$$
\frac{C_{q}(u) C_{q}(u+\lambda)}{A_{q-1}(u+\lambda) A_{q+1}(u)}=1
$$

Eqs. (1.33), (1.38) and (1.43) give a matrix realization of the fusion rules (1.9) which can be re-written as

$$
\left(\tilde{F}^{r}\right)^{2}=\left(I+\tilde{F}^{r-1}\right)\left(I+\tilde{F}^{r+1}\right)
$$

where

$$
\tilde{F}^{r}=F^{r-1} F^{r+1}
$$

\section{Derivation}

Before we go on to study the detailed derivations of (1.33) for the individual torus and cylinder cases, let's study the local properties which are common to both cases.

\subsection{Local properties}

Firstly, let's look at how the product $\boldsymbol{T}_{0}^{q} \boldsymbol{T}_{q}^{1}$ is decomposed into a sum of two terms $\boldsymbol{T}_{0}^{q-1}$ and $\boldsymbol{T}_{0}^{q+1}$ up to scalar factors. 
Because of the vertical push-through property, we can disregard the horizontal fusion projectors and apply them later on as a wrapping of the equation.

The product $\boldsymbol{T}_{0}^{q} \boldsymbol{T}_{q}^{1}$ is realized as two transfer matrices stacked upon each other, the top one being at vertical fusion level 1 and the bottom one at fusion level $q$. Consider an arbitrary column of the torus transfer matrix ( $\zeta$-type seams excluded). In fact, after a simple manipulation (1.60), the product of transfer matrices in the cylinder case will be built up of similar columns. There is a projector $P^{q+1}$ attached to its bottom part, realizing the vertical fusion. The Boltzmann weights of this column can be written in terms of Temperley-Lieb operators,

$$
X_{j+q-1}(v) \ldots X_{j+1}(v+(q-2) \lambda) X_{j}(v+(q-1) \lambda) P_{j+1}^{q+1} X_{j-1}(v+q \lambda)
$$

with $j$ an arbitrary label and $v$ the spectral parameter involved in that particular column, for example $v=u-k \lambda$ for a typical bulk face and $v=u+\xi-k \lambda$ for a face in an $r$-seam. Because an $s$-type seam is the braid limit of an $r$-type seam, we don't lose any generality in considering only $r$-type seams. It is easy to see that the following arguments can be applied also to $\zeta$-type seams and that their contribution is trivial.

We duplicate the projector and insert between its two copies the following identity

$$
\frac{1}{S_{q+1}}\left(S_{q} X_{j}(\lambda)+X_{j}(-q \lambda)\right)=\boldsymbol{I}
$$

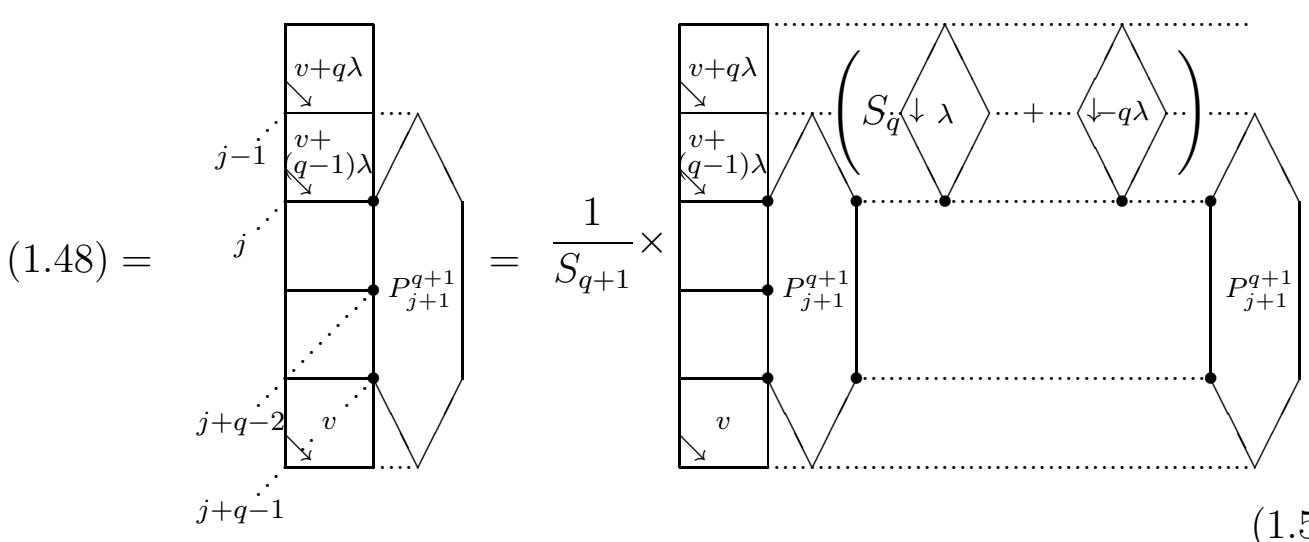




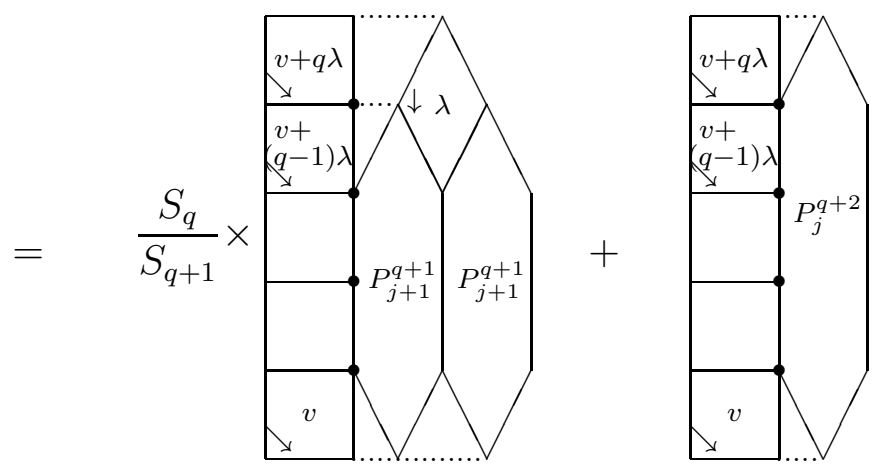

The projector $P_{j}^{q+2}$ in the second term of (1.51) is obtained by the definition (1.4). Thus, this term gives us the column which appears in $\boldsymbol{T}_{0}^{q+1}$ in the functional equation (1.33). By pushing the projector through horizontally in the product of transfer matrices, we can make it appear between every columns and because of the cyclic boundary condition (and a similar argument in the cylinder case), we finally obtain a term which is proportional to $\boldsymbol{T}_{0}^{q+1}$.

We are now going to prove that the first term of (1.51) yields a term proportional to $\boldsymbol{T}_{0}^{q-1}$.

The product $\boldsymbol{T}_{0}^{q} \boldsymbol{T}_{q}^{1}$ involves a whole row of columns such as the LHS of (1.50), hence the columns of Boltzmann weights occur with a fusion projector $P^{q+1}$ between each of them. We can use the push-through property (1.11) to get rid of all but one of these projectors one-by-one:

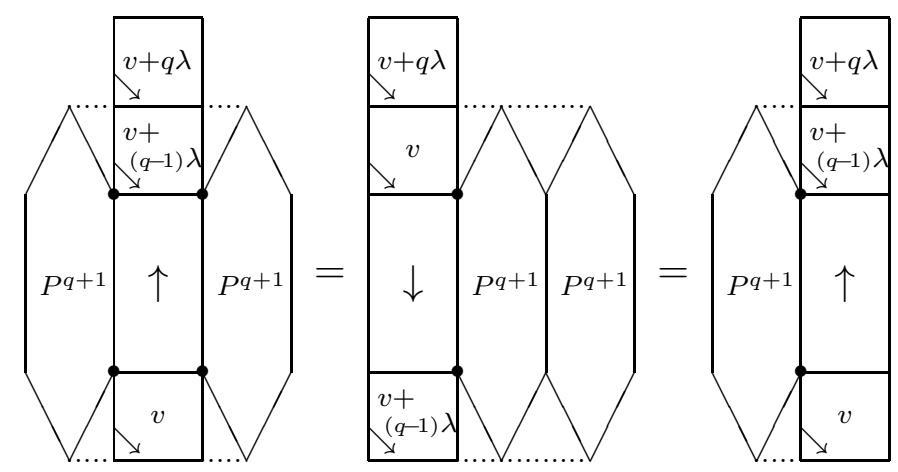

So for a cyclic boundary condition, the projector on the left of the $+\lambda$ face in the first term of (1.51) can be discarded and the one on its left will be the only remaining projector in the row. We will see that the same argument is also valid for the cylindrical boundary condition.

We now make use of the contracting property of the local face projector $X_{j}(\lambda)$ :

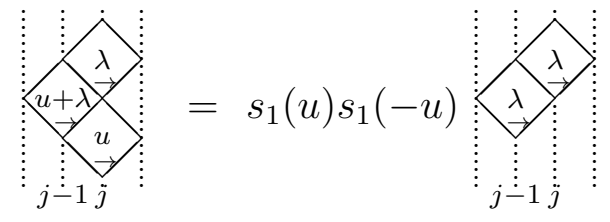


so that the two faces in the top rows of the first term of (1.51) collapse into a scalar under the propagation of the contractor:
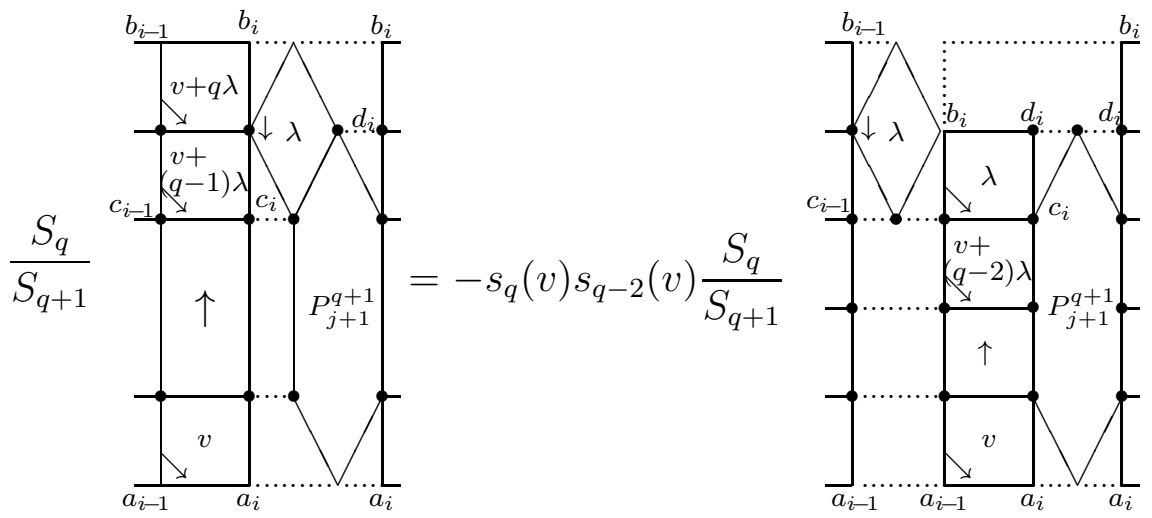

and the newly appeared contractor further collapses the top two faces of the next column on the left. Reapplying the procedure to the rest of the columns on the left and using the cyclic boundary conditions, we finally collapse all of the top two rows. What is left is a scalar contribution $-s_{q}(u) s_{q-2}(u)$ for each column at spectral parameter $u$, a row of faces with spectral parameter $\lambda$ and the local face operator $X_{j}(\lambda)$ at the right of the projector $P^{q+1}$. But the Boltzmann weight of a face at spectral parameter $\lambda$ is simply

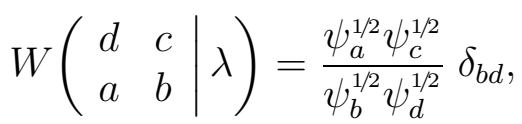

hence the top row disappears, leaving

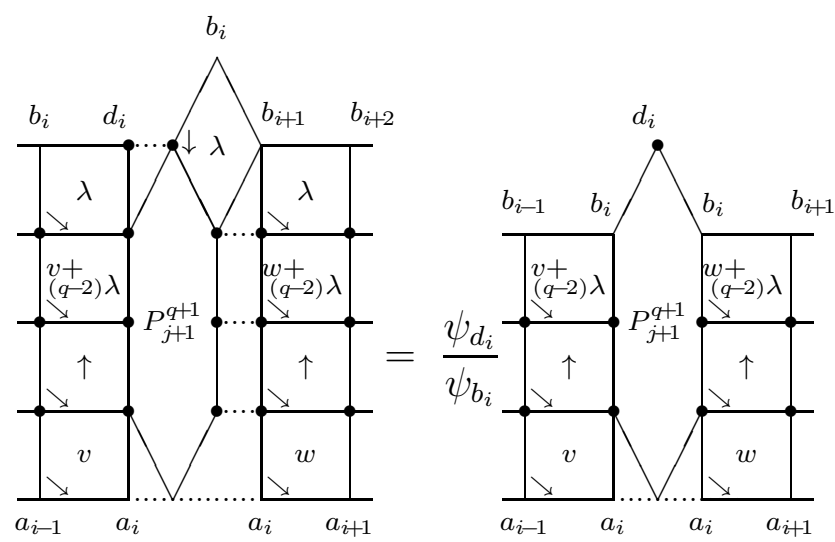

We decompose further the projector $P_{j+1}^{q+1}$ and sum over $d_{i}$ to get the 
shorter fusion projector $P^{q}$ :

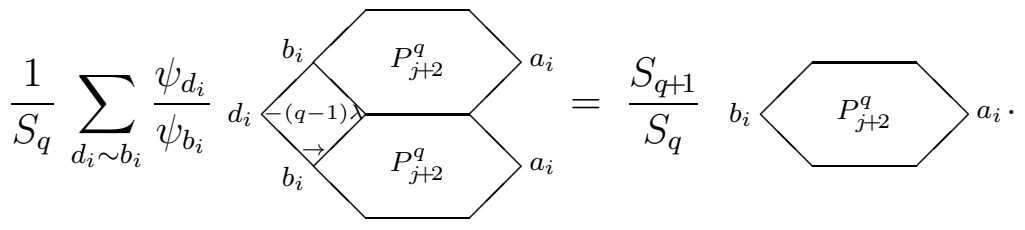

so that (1.54) reduces to the product of the scalar contribution for each column times the matrix valued function $\boldsymbol{T}_{0}^{q-1}$.

We are now going to go into the details of the contribution of each column for each boundary condition.

\subsection{Functional equation on the torus}

Each horizontally $p$-fused bulk column in $\boldsymbol{T}_{0}^{q} \boldsymbol{T}_{q}^{1}$ brings a scalar factor of $s_{q}^{p}(u)$ when collapsed by the contractor. Hence the $N$ bulk faces contribute to the $\boldsymbol{T}_{0}^{q-1}$ term as

$$
f_{q}^{p}(u)=\left[s_{q}^{p}(u)\right]^{N}
$$

The contribution of this same column to the $\boldsymbol{T}_{0}^{q+1}$ term comes from the removal of the common scalar factors which appear in the process of vertical fusion of the top $(p, 1)$-fused face with the larger $(p, q)$-fused face, yielding a $(p, q+1)$-fused face. The result is $f_{q-1}^{p}(u)$.

Likewise, an $r$-type seam contributes in the same proportion but with a shift in the spectral parameter and an adjustment in the common scalar factors, yielding (1.37). It is easily checked that the braid limit of such a factor simply vanishes, hence the $s$-type seams don't contribute to the TBA equation and the same holds for $\zeta$-type seams.

\subsection{Functional equations on the cylinder}

As we discussed in Section 2.3, an $(r, a)$ boundary is the combination of an $r$-seam and a $(1, a)$ boundary so we restrict ourselves to $(1, a)$-boundary conditions.

In the cylinder case, the product $\boldsymbol{T}_{0}^{q} \boldsymbol{T}_{q}^{1}$ of double row transfer matrices is realized as four layers of rows and a typical column is the stack of two $(1,1)$-faces on top of two $(1, q)$-faces, resppectively at spectral parameters $\mu-u+\xi-q \lambda, u+\xi+q \lambda, \mu-u+\xi-(q-1) \lambda$ and $u+\xi$ where $\xi=-k \lambda$ for a usual bulk term involved in a horizontally fused face. Consider the 
following inversion relation (1.14)

$$
\begin{array}{r}
X_{j}^{1 q}(2 u-\mu+(2 q-1) \lambda) X_{j}^{q 1}(-2 u+\mu-(2 q-1) \lambda)= \\
s_{2 q}^{1 q}(2 u-\mu) s_{2-2 q}^{1 q}(-2 u+\mu) P_{j}^{q+1} .
\end{array}
$$

It follows from the GYBE $(1.13)$ that

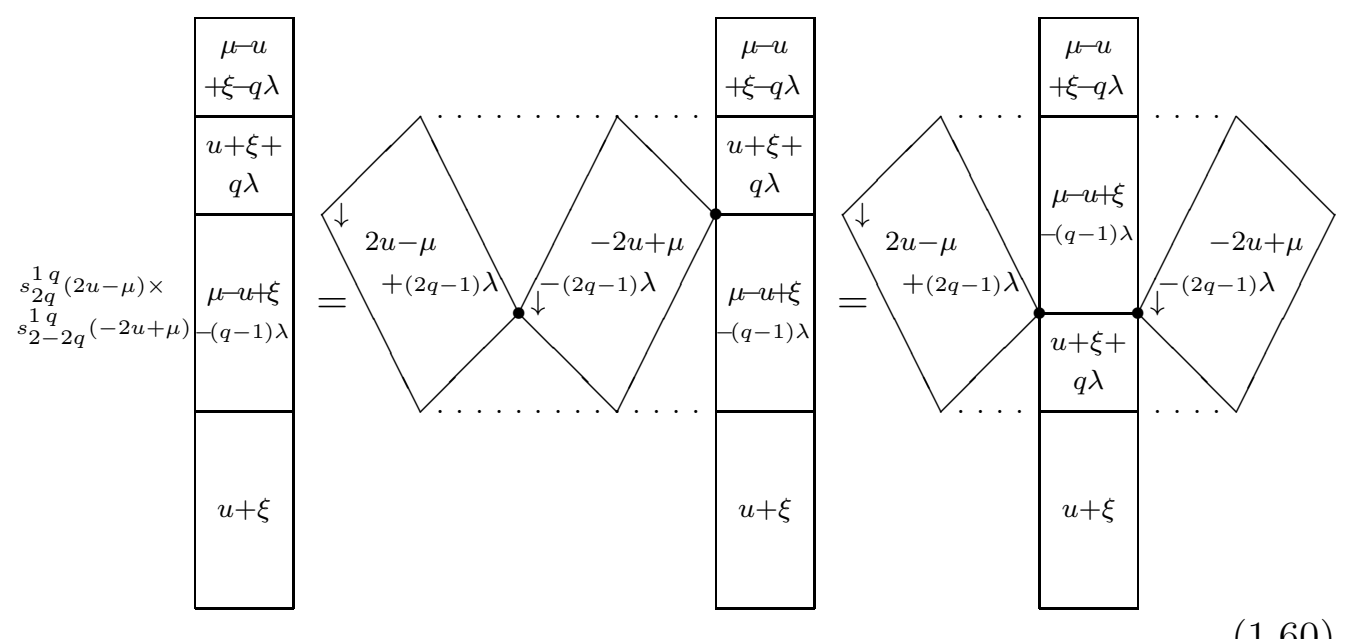

As the rows on the right are of the same structure, the face $X^{q 1}(-2 u+\mu-$ $(2 q-1) \lambda)$ can push through rightward all the way to the right boundary. Similarly, its counterpart $X^{1 q}(2 u-\mu+(2 q-1) \lambda)$ can push through leftwards all the way to the left boundary. Because of Equation (1.28), these rectangular weights, after a crossing symmetry (1.18), simply agglomerate into a larger boundary (minus the larger projector). For a $\left(1, a_{R}\right)$-boundary condition on the right hand side, it reads:

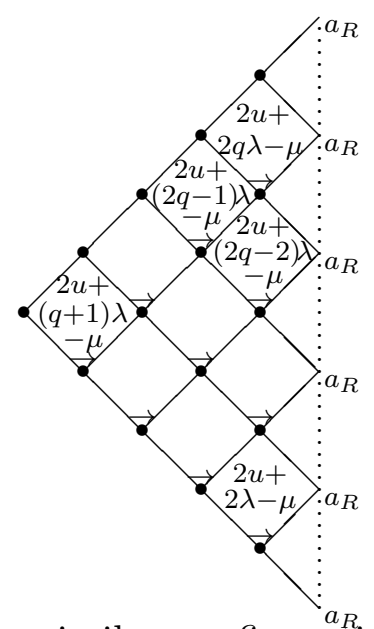

In the bulk, we are now in a similar configuration as in the torus case, simply the columns are doubled. Hence the same technique applies provided the push through of the contractor $X(\lambda)$ and the longer projector $P^{q+2}$ 
behave as expected on the boundary. It is what we are going to discuss now.

It is clear from (1.61) that the push-through property of projectors is still satisfied on the boundary, the projector $P^{q+1}$ coming from the bottom $q$ rows of the product can go up through the right boundary and get back to the $q$ intermediate rows, lower part of the top half. Similarly, coming from the right of these rows, it can go down the left boundary to the lower $q$ rows. Therefore, the term proportional $\boldsymbol{T}_{0}^{q+1}$ proceeds in exactly the same way as in the case of the torus.

Consider now the term $\boldsymbol{T}_{0}^{q-1}$. We need to understand the action of $X_{j}(\lambda)$ on the $\left(1, a_{R}\right)$ right boundary.

Similarly to (1.53), we have

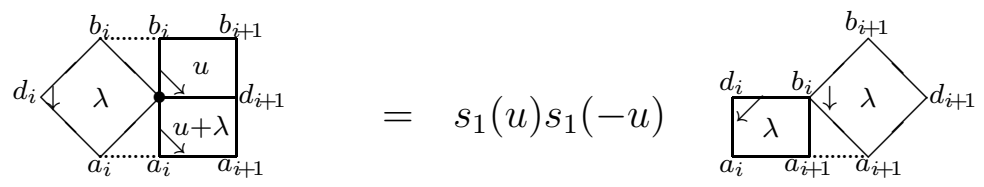

So that when the contractor acts on the $\left(1, a_{R}\right)$ right boundary, we get
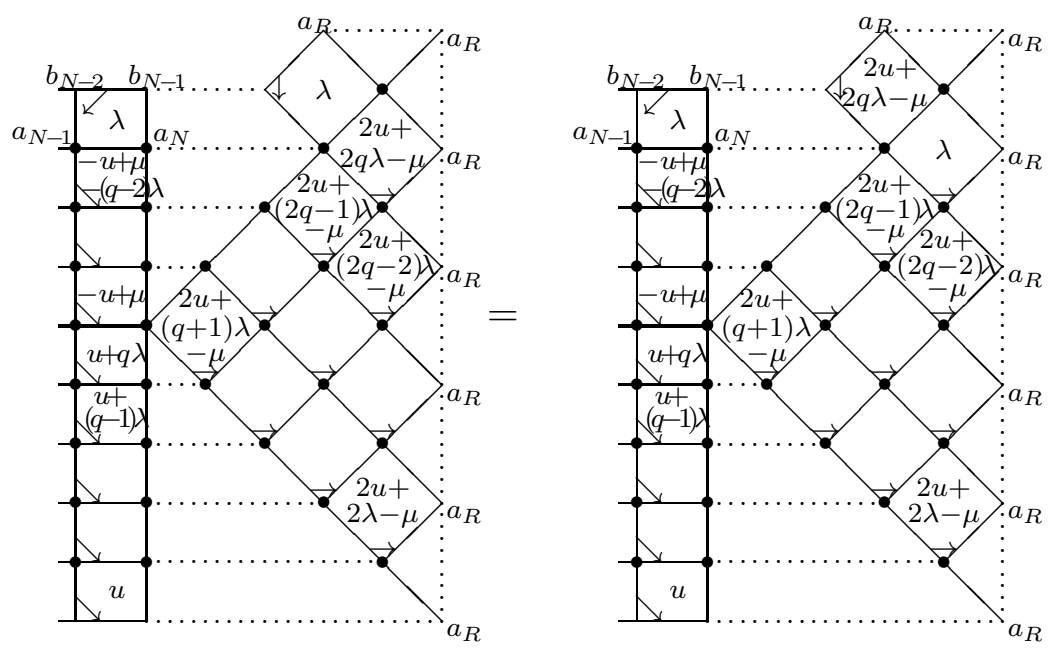

where we have used the crossing symmetry and then the abelian property (1.15) to interchange the parameters $\lambda$ and $2 u+2 q \lambda-\mu$ between two face weights. Then, we apply $(1.53)$ to collapse the faces weights inside the 


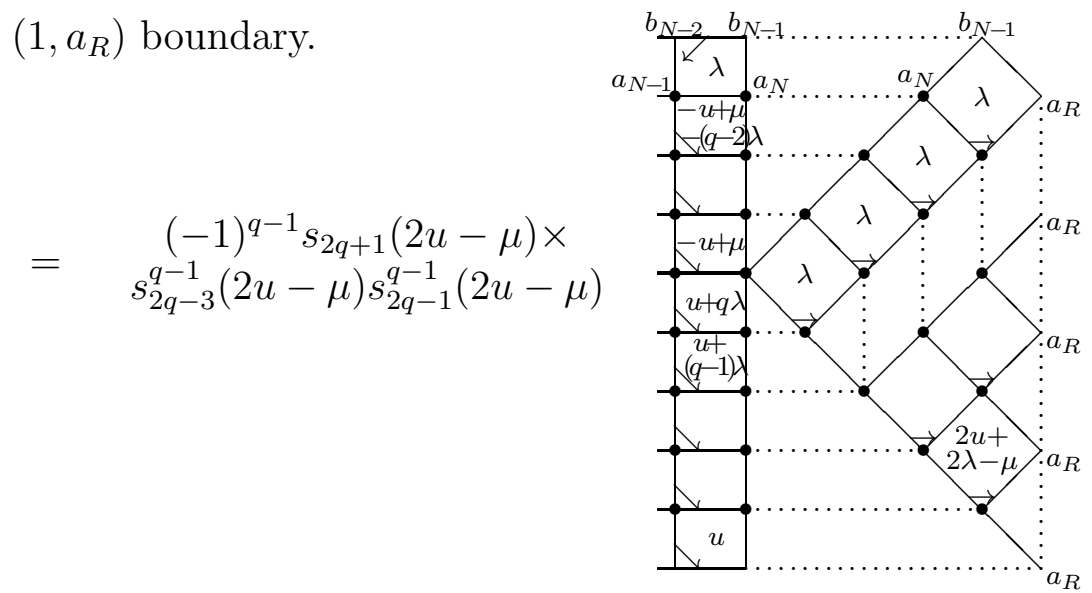

where we use the identity (1.7) to eliminate the face weight with parameter $2 u+2 q \lambda-\mu$.

We can see in (1.64), that the right $\left(1, a_{R}\right)$ boundary of $\boldsymbol{T}_{0}^{q} \boldsymbol{T}_{1}^{1}$ is contracted into a smaller $B_{R}^{q-1}$ under the action of the contractor $X_{j}(\lambda)$. We then continue to collapse the face weights in the bottom half of the transfer matrix with the contractor.

For the $\left(1, a_{L}\right)$ left boundary, the contractor $X_{j}(\lambda)$ acts from the bottom. We rotate the whole diagram by half a turn and we apply the same technique, with scalar factors

$$
(-1)^{q} s_{2 q-3}(2 u-\mu) s_{2 q-2}^{q-1}(2 u-\mu) s_{2 q-4}^{q-1}(2 u-\mu) .
$$

Finally, the contractor can go back to the top row from the left, hence the rest of the proof proceeds as previously.

Collecting the different contributions for all the columns, which come by pair, for the lower and the upper halves of the product $\boldsymbol{T}_{0}^{q} \boldsymbol{T}_{1}^{1}$, with spectral parameters $u+\xi$ and $\mu-u-q \lambda+\xi$ respectively, one gets the result listed in Section 3 .

\section{Discussion}

In this paper we have derived the TBA functional equations for critical lattice models using simple fusion projectors. We point out, however, that the very same functional equations can be derived off-criticality by using the methods of [13]. This applies, for example, for the $A$ and $D$ models which admit elliptic solutions to the Yang-Baxter equations.

We conjecture generally that the form of the TBA functional equations are universal for all integrable lattice models associated with rational CFTs 
and their integrable perturbations. In particular, we expect the known forms [7] of these equations to apply to all integrable boundary conditions.

\section{Acknowledgements}

This research is supported by the Australian Research Council.

\section{References}

[1] Rodney J. Baxter. Partition function of the eight-vertex lattice model. Ann. Physics, 70:193-228, 1972.

[2] Rodney J. Baxter and Paul A. Pearce. Hard hexagons: interfacial tension and correlation length. J. Phys. A, 15(3):897-910, 1982.

[3] Paul A. Pearce. Transfer-matrix inversion identities for exactly solvable lattice-spin models. Phys. Rev. Lett., 58(15):1502-1504, 1987.

[4] V. V. Bazhanov and N. Yu. Reshetikhin. Critical RSOS models and conformal field theory. Internat. J. Modern Phys. A, 4(1):115-142, 1989.

[5] Paul A. Pearce. Row transfer matrix functional equations for $A-D-E$ lattice models. In Infinite analysis, Part A, B (Kyoto, 1991), pages 791-804. World Sci. Publishing, River Edge, NJ, 1992.

[6] Andreas Klümper and Paul A. Pearce. Conformal weights of RSOS lattice models and their fusion hierarchies. Physica A, 183(3):304-350, 1992.

[7] Atsuo Kuniba, Tomoki Nakanishi, and Junji Suzuki. Functional relations in solvable lattice models. I. Functional relations and representation theory. Internat. J. Modern Phys. A, 9(30):5215-5266, 1994.

Atsuo Kuniba, Tomoki Nakanishi, and Junji Suzuki. Functional relations in solvable lattice models. II. Applications. Internat. J. Modern Phys. A, 9(30):5267-5312, 1994.

Atsuo Kuniba and Junji Suzuki. Functional relations and analytic Bethe ansatz for twisted quantum affine algebras. J. Phys. A, 28(3):711-722, 1995. 
1. Integrable Boundaries and Universal TBA Functional Equations

[8] Rodney J. Baxter. Exactly solved models in statistical mechanics. Academic Press Inc. [Harcourt Brace Jovanovich Publishers], London, 1989. Reprint of the 1982 original.

[9] Al. B. Zamolodchikov. Thermodynamic Bethe ansatz in relativistic models: scaling 3-state Potts and Lee-Yang models. Nuclear Phys. B, 342(3):695-720, 1990.

Al. B. Zamolodchikov. Thermodynamic Bethe ansatz for RSOS scattering theories. Nuclear Phys. B, 358(3):497-523, 1991.

Al. B. Zamolodchikov. TBA equations for integrable perturbed $\mathrm{su}(2)_{k} \times \mathrm{su}(2)_{l} / \mathrm{su}(2)_{k+l}$ coset models. Nuclear Phys. B, 366(1):122$132,1991$.

Al. B. Zamolodchikov. From tricritical Ising to critical Ising by thermodynamic Bethe ansatz. Nuclear Phys. B, 358(3):524-546, 1991.

[10] Paul A. Pearce and Bernard Nienhuis. Scaling limit of RSOS lattice models and TBA equations. Nuclear Phys. B, 519(3):579-596, 1998.

[11] Anatol N. Kirillov. Dilogarithm identities and spectra in conforma field theory. In Low-dimensional topology and quantum field theory (Cambridge, 1992), pages 99-108. Plenum, New York, 1993.

A. N. Kirillov. Dilogarithm identities, partitions, and spectra in conformal field theory. Algebra $i$ Analiz, 6(2):152-175, 1994.

[12] E. K. Sklyanin. Boundary conditions for integrable quantum systems. J. Phys. A, 21(10):2375-2389, 1988.

[13] Roger E. Behrend, Paul A. Pearce, and David L. O'Brien. Interactionround-a-face models with fixed boundary conditions: the ABF fusion hierarchy. J. Statist. Phys., 84(1-2):1-48, 1996.

[14] David L. O'Brien and Paul A. Pearce. Surface free energies, interfacial tensions and correlation lengths of the ABF models. J. Phys. A, 30(7):2353-2366, 1997.

[15] David L. O'Brien, Paul A. Pearce, and S. Ole Warnaar. Analytic calculation of conformal partition functions: tricritical hard squares with fixed boundaries. Nuclear Phys. B, 501(3):773-799, 1997.

[16] Roger E. Behrend and Paul A. Pearce. Integrable and conforma boundary conditions for sl(2) $A-D-E$ lattice models and unitary minimal conformal field theories. In Proceedings of the Baxter Revolution 
in Mathematical Physics (Canberra, 2000), volume 102, pages 577-640, 2001.

[17] Christian Mercat and Paul A. Pearce. Integrable and Conforma Boundary Conditions for $\mathbb{Z}_{k}$ Parafermions on a Cylinder. To appear in J. Phys. A, 2001.

[18] Christoph Richard and Paul A. Pearce. Integrable lattice realizations of $N=1$ superconformal boundary conditions. In preparation, 2001.

[19] Roger E. Behrend, Paul A. Pearce, Valentina B. Petkova, and JeanBernard Zuber. Boundary conditions in rational conformal field theories. Nuclear Phys. B, 579(3):707-773, 2000.

[20] C. H. Otto Chui, Christian Mercat, William P. Orrick, and Paul A. Pearce. Integrable and conformal twisted boundary conditions for unitary minimal $A$-D-E models on the torus. hep-th, D0106182, 2001.

[21] V. B. Petkova and J.-B. Zuber. Generalised twisted partition functions. Phys. Lett. B, 504(1-2):157-164, 2001.

[22] Je-Young Choi, Doochul Kim, and Paul A. Pearce. Boundary conditions and inversion identities for solvable lattice models with a sublattice symmetry. J. Phys. A, 22(10):1661-1671, 1989.

[23] C. H. Otto Chui, Christian Mercat, William P. Orrick, and Paul A. Pearce. Integrable and conformal twisted boundary conditions for unitary minimal $A-D-E$ models on the torus. In preparation, 2001.

[24] V.B. Petkova and J.-B. Zuber. The many faces of ocneanu cells. Nuclear Phys. B, 603:449-496, 2001.

[25] Roger E. Behrend and Paul A. Pearce. A construction of solutions to reflection equations for interaction-round-a-face models. J. Phys. A, 29(24):7827-7835, 1996.

[26] Paul A. Pearce and Yu Kui Zhou. Intertwiners and $A-D-E$ lattice models. Internat. J. Modern Phys. B, 7(20-21):3649-3705, 1993. YangBaxter equations in Paris (1992).

[27] Yu Kui Zhou and Paul A. Pearce. Fusion of $A-D-E$ lattice models. Internat. J. Modern Phys. B, 8(25-26):3531-3577, 1994. Perspectives on solvable models. 
1. Integrable Boundaries and Universal TBA Functional Equations

[28] Roger E. Behrend and Paul A. Pearce. Boundary weights for Temperley-Lieb and dilute Temperley-Lieb models. Internat. J. Modern Phys. B, 11(23):2833-2847, 1997. 